\title{
A Comparison of the Autistic Behavior Checklist and the Childhood Autism Rating Scale for Suspected Autism in a Large Chinese Sample
}

Jiahui Chu

Beijing Children's Hospital

Fang Bian

Beijing Children's Hospital

Ruiying Yan

Beijing Children'sHospital

Yanlin Li

Beijing Children's Hospital

Yonghua Cui

Beijing Children's Hospital

Ying Li ( $\square$ liying@bch.com.cn )

Beijing Children's Hospital, Capital Medical University, National Center for Children's Health https://orcid.org/0000-0002-1800-0901

Research article

Keywords: Suspected ASD, CARS, ABC, ROC curve

Posted Date: October 23rd, 2020

DOl: https://doi.org/10.21203/rs.3.rs-54129/v2

License: (9) This work is licensed under a Creative Commons Attribution 4.0 International License.

Read Full License 


\section{Abstract}

Background: Autism is the most common clinical developmental disorder in children. The Childhood Autism Rating Scale (CARS) and Autistic Behavior Checklist (ABC) are the most commonly used assessment scales for diagnosing autism. The aim of this study is to compare the diagnostic validities of CARS and $A B C$ based on a large Chinese sample.

Methods: A receiver operating characteristic (ROC) curve was used to compare the diagnostic validities of CARS and $A B C$, as well as the corresponding cut-off determinations was calculated.

Results: A total of 474 children with suspected autism spectrum disorder (ASD) were screened by CARS and $A B C$. Receiver operating characteristic (ROC) curve analysis was performed and it showed that the area-under-the-curve values of CARS is higher than ABC significantly $(p<0.05)$ and the suggested cutoff values of CARS and $A B C$ were 34 and 67 , respectively.

Conclusion: This study demonstrated that CARS was superior to ABC in terms of its diagnostic validity of assessing suspected ASD cases in children. Based on our results, we recommend the CARS could be used for the assessments for suspected ASD cases in Chinses hospitals.

\section{Background}

Autism is a neurodevelopmental disorder that occurs early in childhood, which results in stereotypical interests, communication deficits, social deficits and repetitive behaviors[1]. Autism spectrum disorder (ASD) has recently received more and more attention, as there have been increasing numbers of young children going to mental health services and community child health centers for screening of ASD in China[2]. Moreover, early diagnosis and intervention play a critical role in the treatment of ASD patients[3]. However, the current approach for the diagnosis of ASD was mainly based on the clinical assessments[46]. The scales most commonly used to diagnose ASD in children are the Autism Behavior Checklist (ABC) and Childhood Autism Rating Scale (CARS)[7].

There are numerous suspected ASD cases (showing one or more symptoms of ASD but no final diagnosis) that originate from community health-service centers and preschools in China, most of whom are initially screened via the Clancy Autism Behavior Scale (CABS)[8]. The cutoff points 14 of CABS was always used as the criteria of suspected ASD in China. When a suspected ASD case was identified, his or her parents will receive the suggestions of going to a hospital for a final diagnosis. When they get to the hospitals for final diagnoses, most of them might undergo further assessments such as ABC or CARS. According to previous studies on various assessments of ASD, CARS exhibits better diagnostic validation compared with that of $A B C[9]$. However, the diagnostic validations and the corresponding cut-offs for CARS and ABC on individuals with suspected ASD were still unclear[4]. Furthermore, for the suspected ASD in China, it remains unclear as to whether CARS is still a better diagnostic tool than ABC. Furthermore, it is unclear whether the current cut-off points for $A B C$ and CARS are suitable for the accurate diagnosis of ASD. 
Therefore, the purpose of this study was to compare the diagnostic validities of CARS and ABC for suspected ASD, as well as to obtain more updated and appropriate cut-off scores for each assessment scale. For the definition of suspected ASD, we still used the CABS as a screening tool with a cut-off score of 14[8]. A receiver operating characteristic (ROC) curve was used to compare the diagnostic validities of CARS and $A B C$, as well as the corresponding cut-off determinations. Our present findings would provide insights into the usage of optimal assessment scales for suspected ASD in Chinese metal health hospitals.

\section{Methods}

\subsection{Participants}

A total of 591 children outpatients from the ASD Unit at Beijing Children's Hospital between June to November of 2019 were identified. First, they were initially screened with CABS. The cutoff points 14 of CABS was always used as the criteria of suspected ASD. Based on this criteria, a total of 117 children outpatients were excluded and 474 were identified as the suspected ASD. Second, the Diagnostic and Statistical Manual of Mental Disorders, Five Version (DSM-5) was used to confirm the diagnosis of ASD via child psychiatrists. A total of 399 children were diagnosed with ASD (Figure 1)(a total of 75 suspected ASD was not meet the criteria of DSM-5). The total sample size included 407 males and 67 females who aged 18 months to 14 years $(4.1 \pm 1.93)$. Then, each included suspected ASD was evaluated by CARS and the $A B C$.

\subsection{Assessment scales}

CARS is one of the most widely used autism assessment scales[10]. It is suitable for children over 18 months old and exhibits good reliability and validity. It is a clinician-rated questionnaire with four frequency levels from 1 to 4 on the basis of observations of individuals and their corresponding information, such as teacher and/or parents reports[11]. CARS is a behavioral rating scale, consisting of 15 items, that is invariably used to quantitatively describe the severity of suspected ASD symptoms[12]. According to the CARS manual, ASD is defined as a CARS score of $\geq 30$ points. A score of 30 or more strongly indicates the existence of ASD. A score of 30-36 suggests mild symptoms, whereas a score of 37 or above suggests moderate to severe ASD[13].

$A B C$ is a well-established assessment scale for screening and diagnosing ASD[7], and it has been used successfully in the differential diagnosis of ASD[14]. The ABC scale contains 57 items segmented into five categories: social and self-help, body and object use, relating to others, language, and sensory features[15]. On the basis of the degree of association with pathological behavior, each item is rated four frequency levels from 1 to 4 . Calculation of the scores for each of the five domains yields the partial and overall scores for each domain[16]. Based on the sum of these scores, severe behavioral characteristics can then be analyzed. Higher scores indicate more autistic behavioral symptoms, In the present study, we used 68 as the ABC cut-off score since this value has been previously recommended by Krug et al.[14]. 
In addition, CABS is the most commonly used screening tool on the Chinese mainland [17]. For this assessment scale, parents complete the Chinese version of CABS, which is based on its first edition in 1969[17]. A total of 14 items are included, each of which has three frequency levels: "Never" (score of 0), "Occasionally" (score of 1), and "Frequently" (score of 2)[8]. In the present study, any participant with a total CABS score $\geq 14$ was identified as a suspected ASD case.

\subsection{Statistical analysis}

The present study used the statistical package, MedCalc19.0, for all statistical analysis. We primarily used ROC curve analysis to determine the best cut-off values for CARS and ABC and to evaluate the sensitivities, specificities, and accuracies of CARS and ABC[10]. ROC curve analysis was also used for comparing diagnostic validations. We also calculated the area under the curve (AUC) for both CARS and $A B C$. Larger AUCs were indicative of improved prediction efficacies. Each cut-off point and its corresponding sensitivity and specificity were also calculated. A p-value $<0.05$ was considered to be statistically significant.

\subsection{Ethical approval}

The ethics committees of Capital Medical University and Beijing Children's Hospital authorized the protocols used in the present study. The Institutional Review Board (IRB) number is 2019-k-396. All of the guardians of the participants offered written informed consent.

\section{Results}

Table 1 presents our assessments of $A B C$ and CARS for suspected ASD. The mean and standard deviation (SD) of CARS total scores were 35.72 and 4.10, respectively, while the mean and SD of $A B C$ total scores were 70.05 and 1.19, respectively. According to the results of t-tests (both $p>0.05$ ), there were no significant differences in CARS or ABC scores between male and female participants. The skewness coefficient and kurtosis coefficient of CARS were 0.99 and 1.39, respectively. In contrast, the skewness coefficient and kurtosis coefficient of $A B C$ were -0.04 and 0.39 , respectively.

The most commonly applied measure of scale reliability is the Cronbach's alpha coefficient ( $a$ ), originally developed by Cronbach (1951), which is used for estimating internal consistency[18]. For this coefficient, larger a values (namely those greater than 0.7 ) are indicative of higher reliability. We found that the Cronbach alpha coefficients of CARS and ABC were 0.772 and 0.426 , respectively (Table 1 ). Therefore, it suggested that the reliability of CARS was higher than that of $A B C$. In addition, we found that the correlation between CARS and CABS was 0.732 .

Next, we performed ROC curve analysis for CARS and ABC, which yielded AUC values of 0.846 and 0.768 , respectively (Figure 2). Notably, ROC curves (AUCs) represent the most commonly applied global index of diagnostic accuracy. The diagnostic capacity of an assessment tool is usually not evaluated by a single number but is instead usually assessed via two or more diagnostic procedures[19]. Diagnosis is generally 
based on a cut-off or threshold value[20]. It is often recommended that the Youden index be used to define the best cut-off point[20]. The cut-off value, which is associated with the maximum of the Youden index, is usually applied as a decision threshold[21]. Table 2 shows the cut-off scores for ABC and CARS with their corresponding sensitivity and specificity values. The results showed that the differences in AUC values and specificities between CARS and $A B C$ were statistically significant $(P<0.05)$. The false-positive rate (1-specificity) was indicative of a lower misdiagnosis rate[19]. We found that the cut-off values of CARS and ABC were 34 and 67, respectively. More details see the Table 2 and Figure 2.

The negative predictive values (NPVs) and positive predictive value (PPVs) of CARS and ABC are shown in Table 3. The PPV for ASD of a screening test is defined as the proportion of children screened as positive who received an ASD diagnosis divided by the total number of screen-positive cases. PPVs and NPVs are affected by the specificity and sensitivity of the screening tool, as well as by the baseline prevalence of ASD in the population being screened[3]. Moreover, we performed a chi-square test on the PPV and NPV values of ABC and CARS, which revealed that there was no significant difference identified between CARS and ABC.

Based on these results, we suggested the diagnostic procedures for suspected ASD was as follow:

\section{Discussion}

In this study, we found that the AUC of CARS was larger than that of ABC. This finding suggests that CARS is better than ABC in terms of its diagnostic validity for suspected ASD. We also found that the cutoff scores of CARS and ABC for suspected ASD were 34 and 67, respectively. Sensitivity and specificity values included in criterion-validity measures are known to be particularly helpful in clinical settings[22]. The results of a t-test on the specificities between these two assessments also revealed a significant difference, indicating that the specificity of CARS was higher than that of ABC. Furthermore, we verified that the Cronbach alpha coefficient of CARS was 0.772 , while that of $A B C$ was 0.426 . This finding suggests that CARS may be more suitable for diagnosing suspected ASD.

CARS is one of the most important tools for the assessment of ASD, such that both clinical and research practices often use it[23]. Recently, CARS-2 was exploited based on the original edition of CARS[24]. CARS-2 (normalized form) is the same as original CARS, whereas CARS-2-HF (high-functioning form) is a newly developed optional diagnostic for evaluating ASD in children over a certain age and with IQ scores above 80[25]. In this study, we revisited the validation of CARS and found that it functioned as a better diagnostic compared with that of ABC. We also identified an updated cut-off score of CARS for its further usage in diagnosing suspected ASD.

One of the advantages of our study is the introduction of the concept of suspected ASD, which differs from concepts offered in previous studies. In China, there are an increasing number of suspected ASD that have been identified at community health-service centers and preschools[17]. It has been reported that early diagnosis plays a critical role for improving outcomes of ASD[26]. In this context, preliminary screening tools are a critical step for timely diagnosis and intervention of ASD[27]. As a preliminary 
screening tool, CABS can help childcare physicians, teachers, and parents to quickly screen children with suspected autism[8].

Moreover, most children with suspected $A B C$ require further assessments, such as via $A B C$ and/or $C A R S$. Based on the results of our present study, we suggest that CARS may be sufficient for further assessment of suspected ASD.

Previous studies have suggested that the cut-off scores of CARS and ABC for distinguishing autism and non-autism are 30 and 68 , respectively[13, 14]. However, for patients with suspected ASD, it has been suggested that these previously proposed cut-off values may no longer be accurate. Based on the results of the present study, we suggest new cut-off value of CARS (namely, a score of 34) for the diagnosis of suspected ASD. Based on our present findings, we suggest that children with suspected ASD be initially screened via CABS and that any suspected cases be further confirmed via CARS.

Based on clinically suspected children with ASD in this present study, we found that the diagnostic validation of CRAS was better than that of $A B C$. Although previous studies have confirmed the strength of CARS, the sample sizes have been limited[8]. In the present study, we confirmed that CARS may be more suitable than ABC for diagnosing ASD in China, especially for suspected ASD[28].

The only available means of ASD diagnosis are behavioral assessments rather than blood tests or noninvasive assessments[29]. Furthermore, in order to conduct the most comprehensive evaluation of ASD, different measurement tools are required in different assessment environments. CARS is a valid and reliable assessment tool that is used for diagnosis and screening ASD in a number of countries[30]. As mentioned above, the main purpose of this study was to explore the diagnostic validation of CARS in a large Chinese sample. Our results further confirmed that CARS is able to effectively and efficiently diagnose patients with suspected ASD. Therefore, in order to comprehensively evaluate ASD, we recommend the combined use of CABS and CARS which might improve the efficiency of clinical works in hospitals.

Three specific limitations were need to be addressed. First, the adult ASD group was not included in this study, future studies should clarify the diagnosis validation of $A B C$ and CARS in different age groups. Second, although a total of 474 outpatients were included in this study, the sample was still small. Large sample of ASD are needed to confirm these results in future studies. Third, CARS-2 has been well developed[31], but there is no Chinese version of CARS-2 currently. More new tools for the assessments of ASD in China were needed, especially the original tools which designed by Chinese researcher in a Chinese setting.

\section{Conclusion}

This study demonstrated that CARS was superior to ABC in terms of its diagnostic validity of assessing suspected ASD cases in children. In the clinical evaluation for suspected ASD, our findings suggest that 
the cut-off values of CARS and ABC were 34 and 67, respectively. Based on our results, we recommend the CARS could be used for the assessments for suspected ASD cases in Chinses hospitals.

\section{Abbreviations}

ASD: Autism Spectrum Disorder; ABC: Autistic Behavior Checklist; CARS: Childhood Autism Rating Scale; ABC: Autistic Behavior Checklist; ROC: Receiver operating characteristic; CABS: Clancy Autism Behavior Scale; AUC: Area Under the Curve; SD: Standard Deviation; NPV: Negative predictive values; PPV: positive predictive value.

\section{Declarations}

\section{Ethics approval and consent to participate}

This study was approved and monitored by the by the Ethics Committee of Beijing Children's Hospital. Written informed consent will be obtained from the participant and/or their guardian before they were included in this study.

\section{Consent for publication}

Not applicable.

\section{Availability of data and materials}

The datasets used and/or analyzed during the current study are available from the corresponding author on reasonable request.

\section{Competing interests}

All of the authors declare that they have no competing interests.

\section{Funding}

This study is supported by the Special Fund of the Pediatric Medical Coordinated Development Center of Beijing Hospitals Authority, No. XTYB201802. Yonghua Cui was the Founder. The funding body had no further role in the study design, the collection, analysis, and interpretation of data, the writing of the manuscript and the decision to submit the paper for publication.

\section{Authors' contributions}

For this manuscript, $\mathrm{YC}$ took the initiative. $\mathrm{FB}, \mathrm{YL}$ and $\mathrm{RY}$ will participated in the data collection. $\mathrm{YL}$ performed the data analysis and $\mathrm{JC}$ finished the draft. $\mathrm{YL}$ and $\mathrm{YC}$ contributed equally for this article. All authors have read and approved the manuscript. 
Acknowledgments

Thanks very much to all participants involved in this study.

Author details

${ }^{1}$ Department of Psychiatry, Beijing Children's Hospital, Capital Medical University, National Center for Children Healthy, Beijing, China.

\section{References}

1. Wan Y, Hu Q, Li T, Jiang L, Du Y, Feng L, Wong JC, Li C: Prevalence of autism spectrum disorders among children in China: a systematic review. Shanghai Arch Psychiatry 2013, 25(2):70-80.

2. Ou JJ, Shi LJ, Xun GL, Chen C, Wu RR, Luo XR, Zhang FY, Zhao JP: Employment and financial burden of families with preschool children diagnosed with autism spectrum disorders in urban China: results from a descriptive study. BMC Psychiatry 2015, 15:3.

3. Zwaigenbaum L, Bauman ML, Fein D, Pierce K, Buie T, Davis PA, Newschaffer C, Robins DL, Wetherby A, Choueiri R et al: Early Screening of Autism Spectrum Disorder: Recommendations for Practice and Research. Pediatrics 2015, 136 Suppl 1:S41-59.

4. LI J-H: Comparison of Clinical Application of Three Children Autism Behavior Rating Scales.Chinese Journal of Contemporary Pediatrics 2005, 7(1):59-62 (In Chinese).

5. El-Ansary A, Bjorklund G, Khemakhem AM, Al-Ayadhi L, Chirumbolo S, Ben Bacha A: MetabolismAssociated Markers and Childhood Autism Rating Scales (CARS) as a Measure of Autism Severity. J Mol Neurosci 2018, 65(3):265-276.

6. Perry A, Condillac RA, Freeman NL, Dunn-Geier J, Belair J: Multi-site study of the Childhood Autism Rating Scale (CARS) in five clinical groups of young children. J Autism Dev Disord 2005, 35(5):625-634.

7. Rellini E, Tortolani D, Trillo S, Carbone S, Montecchi F: Childhood Autism Rating Scale (CARS) and Autism Behavior Checklist (ABC) correspondence and conflicts with DSM-IV criteria in diagnosis of autism. J Autism Dev Disord 2004, 34(6):703-708.

8. Sun X, Allison C, Auyeung B, Matthews FE, Zhang Z, Baron-Cohen S, Brayne C: Comparison between a Mandarin Chinese version of the Childhood Autism Spectrum Test and the Clancy Autism Behaviour Scale in mainland China. Res Dev Disabil 2014, 35(7):1599-1608.

9. Rellini E, Tortolani D, Trillo S, Carbone S, Montecchi F: Childhood Autism Rating Scale (CARS) and Autism Behavior Checklist (ABC) correspondence and conflicts with DSM-IV criteria in diagnosis of autism. J Autism Dev Disord 2004, 34(6):703-708. 
10. Geier DA, Kern JK, Geier MR: A Comparison of the Autism Treatment Evaluation Checklist (ATEC) and the Childhood Autism Rating Scale (CARS) for the Quantitative Evaluation of Autism. J Ment Health Res Intellect Disabil 2013, 6(4):255-267.

11. Saemundsen E, Magnusson P, Smari J, Sigurdardottir S: Autism Diagnostic Interview-Revised and the Childhood Autism Rating Scale: convergence and discrepancy in diagnosing autism. J Autism Dev Disord 2003, 33(3):319-328.

12. Russell PS, Daniel A, Russell S, Mammen P, Abel JS, Raj LE, Shankar SR, Thomas N: Diagnostic accuracy, reliability and validity of Childhood Autism Rating Scale in India. World J Pediatr 2010, 6(2):141-147.

13. Magyar $\mathrm{Cl}$, Pandolfi V: Factor structure evaluation of the childhood autism rating scale. J Autism Dev Disord 2007, 37(9):1787-1794.

14. Yousefı N, Dadgar H, Mohammadi MR, Jalilevand N, Keyhani MR, Mehri A: The Validity and Reliability of Autism Behavior Checklist in Iran. Iran J Psychiatry 2015, 10(3):144-149.

15. Juneja M, Sharma S, Mukherjee SB: Sensitivity of the autism behavior checklist in Indian autistic children. J Dev Behav Pediatr 2010, 31(1):48-49.

16. Marteleto MR, Lima e Menezes CG, Tamanaha AC, Chiari BM, Perissinoto J: Administration of the Autism Behavior Checklist: agreement between parents and professionals' observations in two intervention contexts. Braz J Psychiatry 2008, 30(3):203-208.

17. Sun X, Allison C, Matthews FE, Sharp SJ, Auyeung B, Baron-Cohen S, Brayne C: Prevalence of autism in mainland China, Hong Kong and Taiwan: a systematic review and meta-analysis. Mol Autism 2013, 4(1):7.

18. Sijtsma K: On the Use, the Misuse, and the Very Limited Usefulness of Cronbach's Alpha. Psychometrika 2009, 74(1):107-120.

19. Martinez-Camblor P, Pardo-Fernandez JC: The Youden Index in the Generalized Receiver Operating Characteristic Curve Context. Int J Biostat 2019, 15(1).

20. Hajian-Tilaki K: The choice of methods in determining the optimal cut-off value for quantitative diagnostic test evaluation. Stat Methods Med Res 2018, 27(8):2374-2383.

21. Bantis LE, Nakas CT, Reiser B: Construction of confidence intervals for the maximum of the Youden index and the corresponding cutoff point of a continuous biomarker. Biom J 2019, 61(1):138-156.

22. Trikalinos TA, Balion CM, Coleman Cl, Griffith L, Santaguida PL, Vandermeer B, Fu R: Chapter 8: metaanalysis of test performance when there is a "gold standard". J Gen Intern Med 2012, 27 Suppl 1:S56-66. 
23. Dawkins T, Meyer AT, Van Bourgondien ME: The Relationship Between the Childhood Autism Rating Scale: Second Edition and Clinical Diagnosis Utilizing the DSM-IV-TR and the DSM-5. J Autism Dev Disord 2016, 46(10):3361-3368.

24. Falissard B, Severo CA, Lambert E, Crutel V, Kyaga S, Serret S, Ravel D, Lemonnier E: P.809 Correlation between childhood autism rating scale 2 and clinical global impression improvement. European Neuropsychopharmacology 2019, 29(Supl.6):S538-S539.

25. Moon SJ, Hwang JS, Shin AL, Kim JY, Bae SM, Sheehy-Knight J, Kim JW: Accuracy of the Childhood Autism Rating Scale: a systematic review and meta-analysis. Dev Med Child Neurol 2019, 61(9):10301038.

26. Mayes SD, Calhoun SL, Murray MJ, Pearl A, Black A, Tierney CD: Final DSM-5 under-identifies mild Autism Spectrum Disorder: Agreement between the DSM-5, CARS, CASD, and clinical diagnoses. Research in Autism Spectrum Disorders 2014, 8(2):68-73.

27. Mukherjee SB, Malhotra MK, Aneja S, Chakraborty S, Deshpande S: Diagnostic accuracy of Indian Scale for Assessment of Autism (ISAA) in chidren aged 2-9 years. Indian Pediatr 2015, 52(3):212-216.

28. Park EY, Kim J: Factor structure of the Childhood Autism Rating Scale as per DSM-5. Pediatr Int 2016, 58(2):139-145.

29. Norris M, Lecavalier L: Screening accuracy of Level 2 autism spectrum disorder rating scales. A review of selected instruments. Autism 2010, 14(4):263-284.

30. Incekas Gassaloglu S, Baykara B, Avcil S, Demiral Y: Validity and Reliability Analysis of Turkish Version of Childhood Autism Rating Scale. Turk Psikiyatri Derg 2016, 27(4):266-274.

31. Schopler E, Wellman GJ, Love SR: Childhood Autism Rating Scale. 2nd edn. 2010:Torrance, CA: Western Psychological Services.

\section{Tables}

Table 1

The Description of $A B C$ and CARS

\begin{tabular}{|llllllll|}
\hline Variables & Mean & SD & Kurtosis & Skewness & Cronbach's a & AUC & AUC (95\%Cl) \\
\hline ABC & 70.05 & 1.19 & 0.39 & -0.04 & 0.426 & 0.768 & $0.727 \sim 0.805$ \\
\hline CARS & 35.72 & 4.10 & 1.39 & 0.99 & 0.772 & 0.846 & $0.810 \sim 0.877$ \\
\hline
\end{tabular}

Note: ABC: Autistic Behavior Checklist; CARS: Childhood Autism Rating Scale; AUC: Area Under Curve; $\mathrm{Cl}$ : confidence interval. 
Table 2

The cutoff points and corresponding sensitivity and specificity of $A B C$ and CARS

\begin{tabular}{|c|c|c|c|c|c|c|}
\hline Criterion & Sensitivity & $95 \% \mathrm{Cl}$ & Specificity & $95 \% \mathrm{Cl}$ & +LR & -LR \\
\hline \multicolumn{7}{|l|}{$A B C$} \\
\hline \multirow{2}{*}{$>62$} & 80.45 & $76.2-84.2$ & 58.67 & $46.7-69.9$ & 1.95 & 0.33 \\
\hline & 77.94 & $73.6-81.9$ & 61.33 & $49.4-72.4$ & 2.02 & 0.36 \\
\hline \multirow{2}{*}{$>63$} & & & & & & 0.00 \\
\hline & 76.19 & $71.7-80.3$ & 65.33 & $53.5-76.0$ & 2.20 & 0.36 \\
\hline \multirow{2}{*}{$\begin{array}{l}>64 \\
>65\end{array}$} & 73.68 & $69.1-77.9$ & 68.00 & $56.2-78.3$ & 2.30 & 0.39 \\
\hline & 72.18 & $67.5-76.5$ & 69.33 & $57.6-79.5$ & 2.35 & 0.40 \\
\hline$>66$ & 68.17 & $63.4-72.7$ & 76.00 & $64.7-85.1$ & 2.84 & 0.42 \\
\hline$>67$ & 63.41 & $58.5-68.1$ & 80.00 & $69.2-88.4$ & 3.17 & 0.46 \\
\hline$>68$ & 58.90 & $53.9-63.8$ & 82.67 & $72.2-90.4$ & 3.40 & 0.50 \\
\hline$>69$ & 48.12 & $43.1-53.1$ & 82.67 & $72.2-90.4$ & 2.78 & 0.63 \\
\hline$>72$ & 44.36 & $39.4-49.4$ & 84.00 & 73.7-91.4 & 2.77 & 0.66 \\
\hline$>73$ & 41.10 & $36.2-46.1$ & 85.33 & $75.3-92.4$ & 2.80 & 0.69 \\
\hline$>74$ & 92.98 & $90.0-95.3$ & 40.00 & $28.9-52.0$ & 1.55 & \\
\hline CARS & 85.46 & $81.6-88.8$ & 60.00 & $48.0-71.1$ & 2.14 & 0.18 \\
\hline \multirow{2}{*}{$\begin{array}{l}>30 \\
>31\end{array}$} & 78.70 & $74.3-82.6$ & 76.00 & $64.7-85.1$ & 3.28 & 0.24 \\
\hline & 68.42 & $63.6-73.0$ & 90.67 & 81.7-96.2 & 7.33 & 0.28 \\
\hline$>32$ & 57.64 & $52.6-62.5$ & 94.67 & $86.9-98.5$ & 10.81 & 0.35 \\
\hline$>33$ & 33.33 & $28.7-38.2$ & 97.33 & $90.7-99.7$ & 12.50 & 0.45 \\
\hline$>34$ & 29.07 & $24.7-33.8$ & 98.67 & $92.8-100.0$ & 21.80 & 0.68 \\
\hline$>35$ & 1.00 & $0.3-2.5$ & 98.67 & $92.8-100.0$ & 0.75 & 0.72 \\
\hline$>36$ & & & & & & 1.00 \\
\hline$>37$ & & & & & & \\
\hline
\end{tabular}

Note: ABC: Autistic Behavior Checklist; CARS: Childhood Autism Rating Scale; Cl: confidence interval; +LR: Positive Likelihood Ratio; -LR: Negative Likelihood Ratio.

Table 3

The PPV and NPV for ABC and CARS

\begin{tabular}{|lllll|}
\hline Variables & ABC & CARS & Chi-square value & P-value \\
\hline PPV & $93 \%$ & $95 \%$ & 1.048 & 0.306 \\
\hline NPV & $32 \%$ & $40 \%$ & 2.243 & 0.134 \\
\hline
\end{tabular}

Note: ABC: Autistic Behavior Checklist; CARS: Childhood Autism Rating Scale; PPV: Positive Predictive Value; NPV: Negative Predictive Value. 


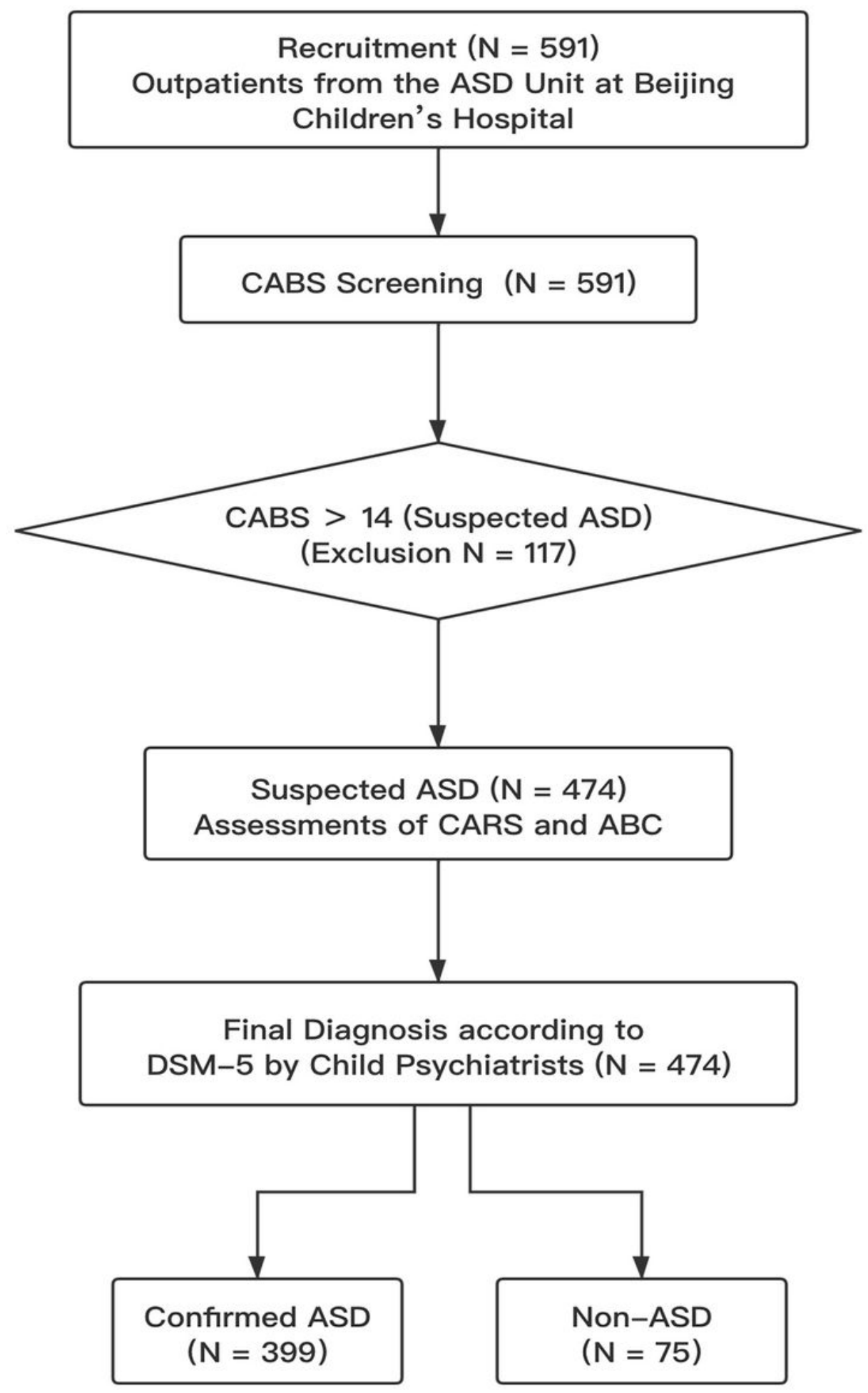

Figure 1

Flowchart of the recruitment of participants in the present study 


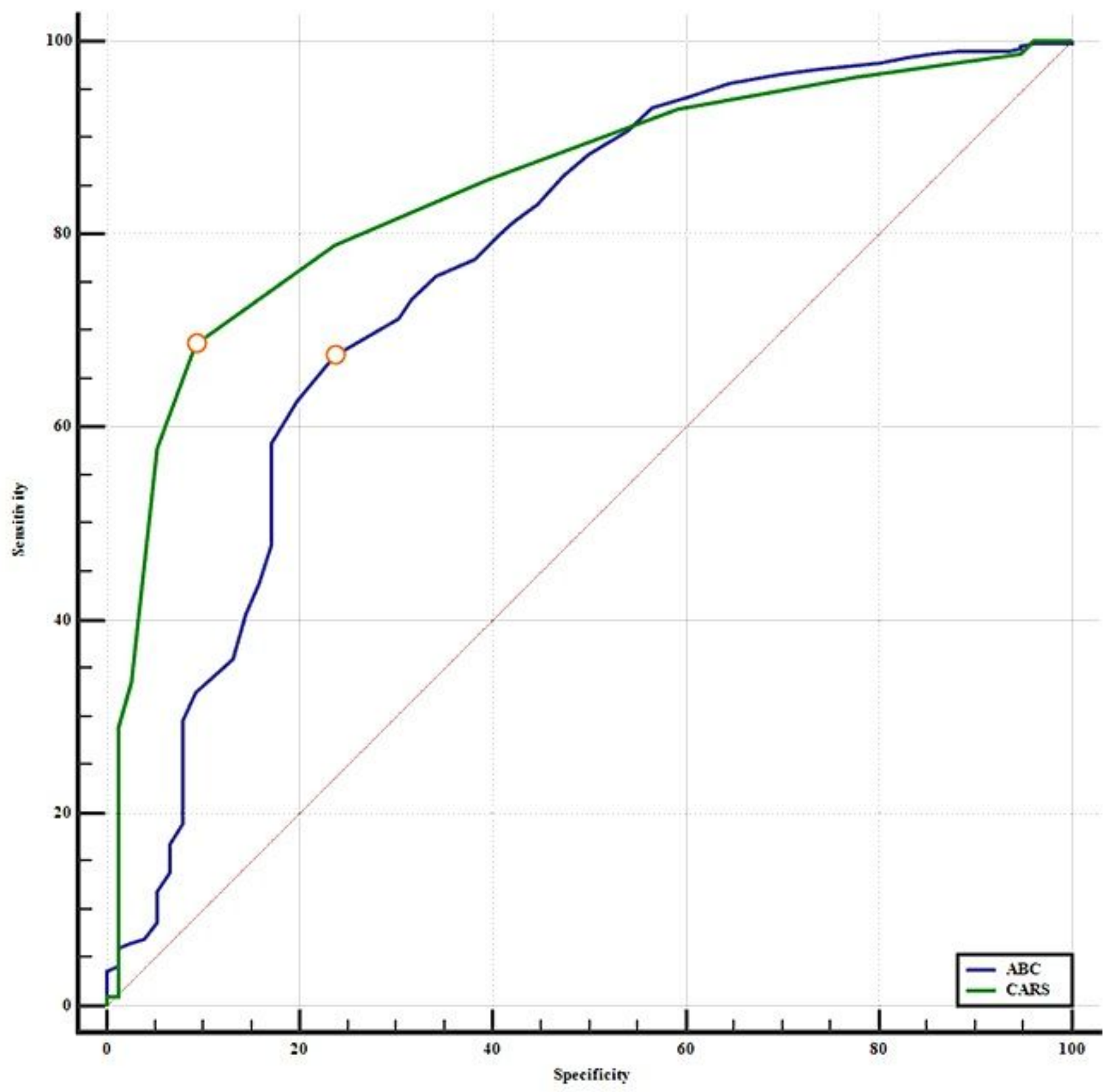

Figure 2

ROC curves of $A B C$ and $C A R S$. 


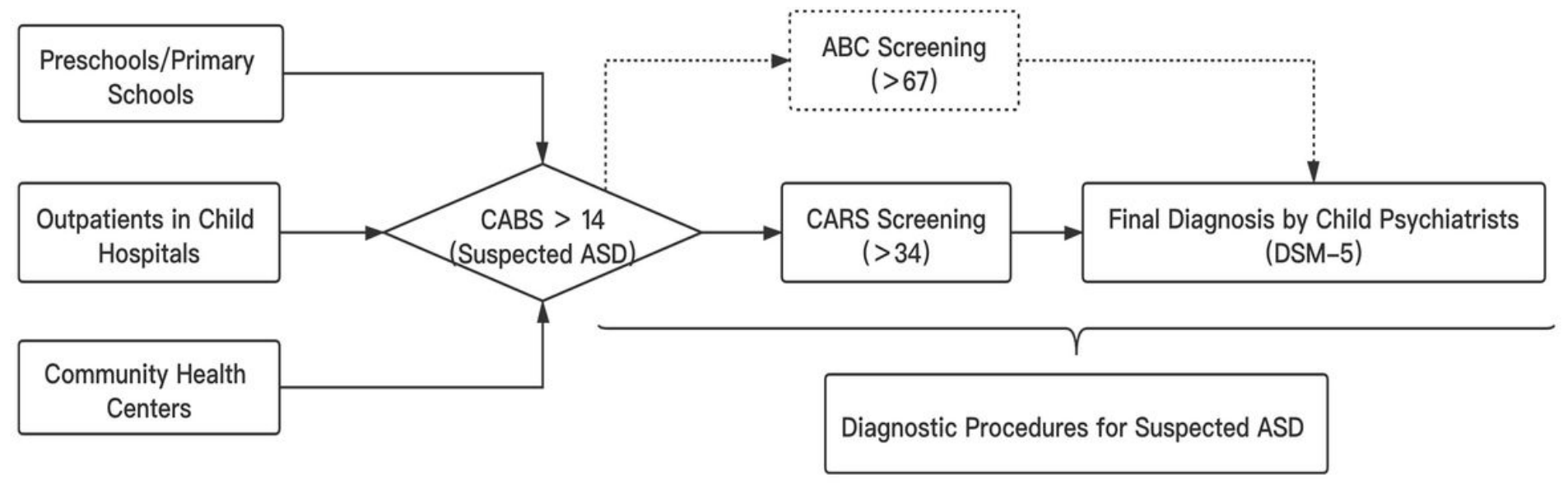

Figure 3

The suggested diagnostic procedures of suspected ASD

\section{Supplementary Files}

This is a list of supplementary files associated with this preprint. Click to download.

- Data.xlsx 